\title{
A Modern Reading of the 40 Principles of TIPS Using the Example of the Technology for Activation of Fuel Mixtures
}

\author{
Valentin Korobov \\ Master of Management, Advisor of the Russian Academy of Natural History, \\ Member of the New York Academy of Sciences, PhD
}

\begin{abstract}
The reader of this article is able to not only familiarize himself/herself with a range of actual versatile innovative inventions but also to be wrapped up in the principles of invention creation process and explore an issue of the scientific background lagging in terms of modern demands and approaches to invention
\end{abstract}

Key words: fuel, mixtures, invention, technology

\section{Technology for activation of fuel mixtures}

Various technologies and devices for achievement of the final goal that is reduction of the fuel consumption in the transport vehicle engines are known at present.

The technology offered for exerting impact on the dynamically moving fuel flow in the fuel pipeline includes several stages of deep hydrodynamic and aerodynamic influence on the fuel.

Conditions for operation of Bernoulli's principle in the pipeline are created to increase impact intensity and decrease or eliminate additional energy expenditures for impact, thus allowing to obtain highly-efficient fuel mixture with minimal energy expenditures at the final stage of aerodynamic impact and without any expenditures at the hydrodynamic impact stage and significantly increase efficiency of the combustion process and at the same time to decrease specific fuel consumption rate.

A device for implementation of the technology offered has two design variants. The first design is used for cases when the fuel has at least two different components (for instance, gasoline and water) or when the fuel has at least three different components (for instance, gasoline, ethanol and water). The second design variant is designated for cases when the fuel is consisted of just one component (for instance, gasoline).

The device of all designs includes a hydrodynamic mechanism for turbulence homogenization connected with the pump for delivery of fuel from the fuel tank and an aerodynamic foaming flow activator connected to a mini-compressor having a rotation drive from the kinematic branch circuit of the engine shaft and through a fuel pipeline with an outlet 
to the multiflame fuel spray nozzles integrated into the pipeline and connected functionally and by design with the help of hydromechanical and aeromechanical interface.

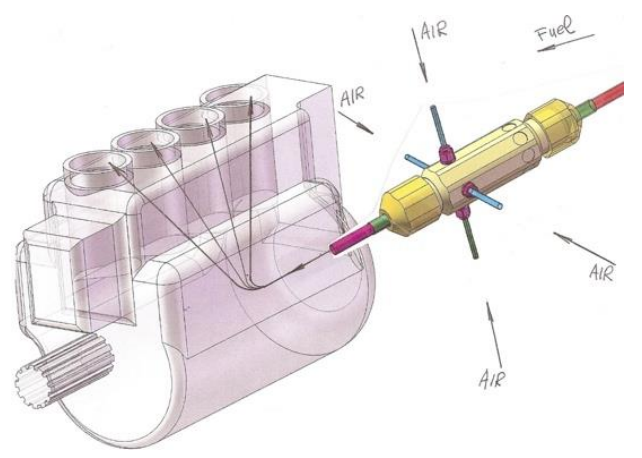

Fig. 1 - The Figure shows a 3D model of the engine with fuel activation device

The fuel is activated by means of homogenous mixing of liquid fuel with microscopic air bubbles. The mixing takes place in the pipeline during passing of liquid fuel from the low pressure fuel pump to the high pressure fuel pump. And the air is supplied from four sides to the device area where a depression zone is formed during fuel movement through coaxial channels of the device and in some cases vacuum is formed in this zone.

According to this mixing option, air bubble sizes do not exceed several micrometers and thickness of the external spherical membrane of each bubble does not exceed several micrometers, as well. After injection the air bubbles get broken and correspondingly the membranes get broken as well, thus significantly increasing dispersity of the fuel particles in the combustion chamber.

\section{TIPS and AIPS}

The Algorithm for Inventive Problem Solving (AIPS) is a step-wise program (sequence of actions) for detection and solution of discrepancies i.e. inventive problem solving (consisting of approximately 85 steps). AIPS includes the program itself:

- $\quad$ software supplied in the result of data collection;

- methods for control of psychological factors being an integral part of the creative thinking development (ㄷD) methods.

There are the other approaches helping an inventor to unlock his/her creative potential. Major part of these methods is heuristic. They all are based on the psychology and logics and neither of them qualify for being a scientific theory (as opposed to TIPS):

1. Trial-and-error method; 


\section{Brainstorming;}

3. Morphological analysis;

4. Focal object method;

5. Test question method;

TIPS - Theory for Inventive Problem Solving.

Modern TIPS consists of several schools developing the conventional TIPS and adding new sections absent in the classical version.

Thoroughly developed a technological core of TIPS (methods, AIPS, substantial and field analysis) remains almost unchanged and the activity of modern schools is mainly aimed at rethinking, restructuring and promotion of TIPS that means it has rather philosophical and promotional than technological nature.

In this connection modern schools of TIPS frequently integrate and adapt digital technologies and the newly emerged due to them possibilities at all the stages of project development. It is important to note that nowadays TIPS is actively used in the field of advertising, business, art, early learning, etc. though initially it was designated for predominantly technical creativity.

Conventional TIPS is a common technical version. Multiple special-purpose versions of TIPS notable for their nomenclature and extent of data collection are required for practical use in the field of engineering.

A Soviet patent engineer, writer and scientist Heinrich Altschuller was convinced in possibility to reveal based on the predecessors' experience repeated methods of successful inventions and possibility to teach this technique all the interested and capable for learning.

A study of over 40 thousand author's certificates and patents was carried out and based on the patterns of technical systems and invention methods revealed, the Theory of Inventive Problem Solving (TIPS) was developed.

But at present, the task and methods for its solution are principally different, as in the system of such patterns and their interrelation, new composite materials, mobile applications and programmable controllers, control processors with artificial intelligence elements and smart engineering and technological solutions allowing to adapt new engineering and technological interrelations including programmable solutions have emerged despite the fact 
that purely programmable solutions are not engineering ones and they are not correspondingly classified as inventions.

When an inventor faces an engineering problem for the first time and it is peculiarly characteristic to the start of any project, it is usually quite unstructured and does not contain any instructions for solution ways.

Besides, a startup company, as a rule, does not have any production experience and equipment to be used for even some experiments and tests.

Such form of a problem and such form for setting an initial task are called an invention situation in TIPS. It is main disadvantage is that the engineer has too many ways and methods for solution. It is quite labor-consuming and expensive to handle them all and the selection of the way at random leads to inefficient trial-and-error method.

Therefore, the first step on the way to an invention is to restate the situation in such a way as the statement itself cuts all the prospectless and inefficient ways for solution. And in this case we have another question what solutions are effective and what are not?

H. Altschuller supposed that the most effective solution to the problem is that is reached in itself but only due to the resources available. Thus, he arrived at the wording of an ideal final result (IFR): "Some element (X element) of the system or environment itself eliminates adverse effect keeping an ability to produce effective action".

Having obtained a tool for screening of inefficient solutions, the invention situation can be reformulated to a standard mini task: "according to IFR everything should remain unchanged but either harmful, unnecessary property should be eliminated or new, useful property should be formed". A key idea of the mini task is to avoid significant (and expensive) changes and first of all consider the simplest solutions.

It is commonly known that the TIPS makes out 3 types of discrepancies (ordered by level of the solution difficulty):

- an administrative discrepancy: "the system should be improved but I do not know how (unable or not entitled) to do this". This discrepancy is the simplest and can be eliminated by either improvement of additional materials or making/ revoking administrative decisions. In the modern world far too many discrepancies of administrative nature are those connected with the commercial conditions and criteria for implementation of the future invention taken as a technology basis of the innovative product; 
- an engineering discrepancy: "improvement of one system parameter results in worsening of the other". The engineering discrepancy is the setting of an inventive problem itself. Transfer from the administrative discrepancy to the engineering one decreases dimension of the problem, narrows the field for solution search and allows going from the trial-and-error method to the algorithm for solution of the inventive problem that either offers using one or several standard engineering methods or (in case of challenging problems) reveals one or several physical discrepancies. At present, in the context of engineering discrepancies, it is necessary to see the impact of the commercial conditions for implementation of the innovative product exerted to them;

- a physical discrepancy: "in order to improve the system some definite part of it should be in various physical states at the same time that is impossible". Physical discrepancy is the most fundamental as an inventor faces the restrictions associated with the physical laws of nature. The inventor should use the physical effect guide and the table for their use to solve the problem.

The analysis of many thousands of inventions has allowed finding out that with all the variety of engineering discrepancies, most of them are solved by 40 main methods. The works on compiling of such method list was already started by H. S. Altschuller at the early stages of the Theory of Inventive Problem Solving establishment.

The system of methods used in TIPS includes both simple methods and the double ones correlating with them as integrated with program digital technologies used at all the stages of design, production and operation.

Simple methods allow solving engineering discrepancies. The most popular among the simple methods are 40 main methods described below.

\section{Modern reading of the $\mathbf{4 0}$ principles of the TIPS}

So let's get back to the topic of the Theory of Inventive Problem Solving and consider 40 known principles in terms of the use of methods and techniques inherent in and characteristic to the smart production, smart transport, smart design, etc.

1. Segmentation principle:

- to divide an object into independent segments;

- to make the object divisible;

- to increase the object segmentation degree. 
In the modern machines and devices, it is almost impossible to fulfill the criterion for independence of the segments into which the object is recommended to be divided.

If take into account the fact that modern innovation objects are often an integrative combination of the device, system, program and method, it will be definitely clear that all the parts or components of the object are all more or less tied up in these elements.

If the logic is followed, then it turns out that in case complete autonomy and independence of the object components is to be reached, it is necessary to make each component compliant with the specified device, system, program and methods that, considering the requirements of patent law on indivisibility of the invention subject and knowing the design principle on pointlessness of repeating all the design, program, technological and algorithmic features discriminated in the process of the object segmentation in each of the segments, makes this method rather harmful than useful.

The methods of modern innovative design is a large-scale integration and segmentation principles shall be most likely transformed into the principle for selection of the autonomous functional components of the object for horizontal and vertical layout integration.

As there is no pure invention in the modern innovation process then at least one of the integration elements shall be the principle of commercial feasibility for horizontal integration and the principles of implementation versatility in the various technological categories not always at first logically interconnected for vertical integration.

The Theory of Inventive Problem Solving and the Algorithm for Inventive Problem Solving have been developed at the time and under conditions when in any case in the Soviet economic system there was no production of the versatile standardized structural and technological components.

This defines a principal difference of the modern machine design and the design, methods and engineering criteria existed at the time of the Theory of Inventive Problem Solving and the Algorithm for Inventive Problem Solving development.

First of all, new principles of machine design inherent in smart engineering solutions in the sphere of transport and transport vehicles shall be defined.

At present, the most significant components of this system of interconnected engineering solutions and the software products and mobile applications associated with them are in particular an integrative concept for the application of software products in the 
technological cycle of basic engineering objects primarily inherent in transport vehicles such as the engines of all types, fuel technologies, environmental systems and control of their combinations in the real time mode.

2. Bracketing out principle:

- to separate an interfering part (an interfering property) from the object;

- to separate a single required part (required property).

The bracketing out is a function of computer simulation in the real time mode by using a mobile application integrated into the specified transportation system as well in the smart transportation systems.

The results of such simulation allow to significantly approximate the operation modes of transportation system and each transport vehicle included to the systems containing the artificial intelligence elements.

3. Local quality principle:

- to move from the homogenous object structure (or external environment, external impact) to the non-homogenous one;

- various parts of the object shall have (fulfill) various functions;

- each part of the object shall be under conditions the most favorable for its operation.

The most favorable operation mode in such system is defined based on the results of computer simulation of the optimal model for smart transportation system and can be transformed for each transport vehicle included in this system.

4. Asymmetry principle:

- to move from a symmetrical object form to the asymmetrical one;

- to increase the asymmetry level, if the object is asymmetrical.

This principle allows keeping independence of each transport vehicle included in the smart transportation system and ensures maximally possible level for integration of each transport vehicle into the common tasks and characteristics of the smart transportation system.

5. Combining principle:

- to combine homogenous or designated for related operations objects;

- to combine the operations homogenous or related as to execution time.

All the components of smart transportation system form an aggregate of smart transport vehicles and each of them separately and all together controlled or regulated by a mobile 
application can unite the transport vehicles executing the homogenous operations or separate the transport vehicles or their groups executing non-homogenous operations but the homogeneity or non-homogeneity level is defined by means of the mobile application.

6. Versatility principle:

- the object executes several different functions and owning to this there is no necessity in the other objects.

In this case an issue of appropriateness of the object fulfilling the functions inherent in the other objects becomes obvious and relevant. This tool can be used only by one operator and in case it is necessary to simultaneously use a new function of the device, then the other tool shall be available meaning that this device is commercially of no use and will not be supported by the investors.

This principle has quite different format in the complex smart systems where a combining function is first of all executed by a certain mobile application solving complex tasks for control, monitoring and measurement and at the same time containing all the necessary provisional information for prompt computer simulation in the real time mode.

7. "Stacking doll" principle:

- one of the objects is located inside the other that in its turn is placed inside the third object, etc.;

- one object is brought through the cavities in the other object.

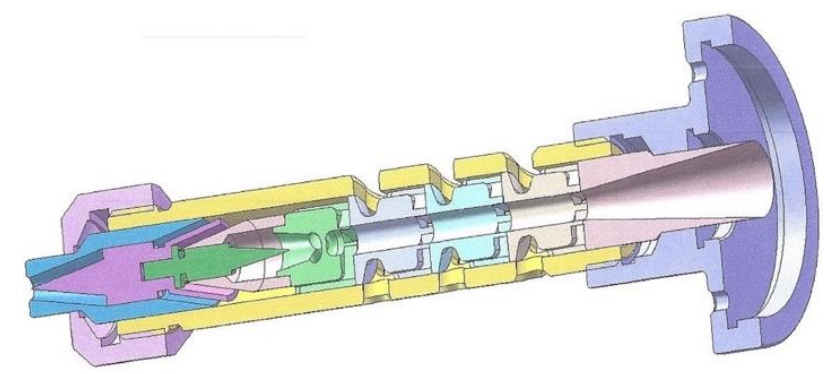

Fig. 2 - Actual structure of the fuel mixture activation device in the carburetor engines built according to the "stacking doll" principle

Design principles are similar to the general principles for design of the device for activation of fuel mixtures and characterized with the use of an external device body as a gauge defining the position of each internal element.

All the internal cavities and channels of one component are connected with the same channels and cavities of related components and the structural elements of vortex generators 
are connected both geometrically and functionally with the suction openings of external body meaning that there are all the signs of a coaxial "stacking doll" and in this particular case determining quality and efficiency of the whole device as applied to the system conditions of higher level in its turn providing for compliance with the requirements of smart transportation systems.

8. Counterweight principle:

- to compensate the object weight by means of combining with the other object of elevating force;

- to compensate the object weight by means of interaction with the environment (due to aerodynamic and hydrodynamic forces).

In this case under the modern conditions there can be variants connected with the potential use of composite material range of various specific weights.

Today's methods and programs of machine engineering allow real time simulation of the weight of any part of the structure with analysis of related structural element reaction to the weight characteristics of the interacting parts and their mutual influence on the adjacent units and joints.

9. Preliminary counteraction principle:

- to strain the object in advance and the strains shall be opposite to the inadmissible or unfavorable operating strains;

- if it is necessary to do some action according to the task provision, counteraction shall be done in advance.

When this principle was defined and classified for the first time, there were no capabilities for computer simulation that often resulted in execution of many actions and operations that nowadays in many cases are automatically done at the design stage by means of actual standard computer engineering programs.

With the help of these programs actions and engineering variants are optimized during design and the most optimal variant with regard to all the circumstances and initial technical requirements is included in the project.

10. Preliminary action principles:

- to do the required action in advance (to the fullest extent or partially); 
- to place the objects in advance in such a way as they can be operated without time expenditures for delivery and from the most convenient point.

As the activation device does not include movable parts, its operating cycle is particularly execution of the actions without time expenditures for positioning of the device components.

11. "A foreseen danger" principle:

- to compensate relatively low reliability of the object with the emergency facilities prepared in advance.

In terms of modern relations between investors and inventors it is clear that the low reliability of the object will definitely and for sure prevent this object from being marketed.

Compensations indeed do not compensate anything and only reliable and absolutely operative structure defines actual commercial success of the object.

12. Principle of equipotentiality:

- To change the conditions of operation so that the object is neither lifted up nor lowered.

The absence of movable parts in the device construction confirms complete correspondence to that principle.

13. Principle of "vice versa":

- To perform the opposite action instead of the action imposed by the conditions of the task;

- To design the traveling part of the object or outer medium immovable, and the immovable one as traveling;

- To turn the object upside down, to turn it inside out.

This is the principle implemented in the device for activating composite fuels, everything is vice versa - activation takes place without using movable parts.

14. Principle of spherocity:

- To proceed from straightforward parts to curvilinear ones, from flat surfaces to spherical ones, from cube-shaped and parallelepiped-shaped parts to spherical constructions;

- To use rollers, balls, spirals;

- To proceed from rectilinear motion to rotational motion, to use the centrifugal force.

Using only cylindrical parts as an equivalent of the principle.

15. Principle of responsiveness: 
- Characteristics of the object (or outer medium) must be changed to be optimal at every stage of operation;

- To divide the object into parts able to travel relative to each other;

- If the object is immovable as a whole, to make it movable, traveling.

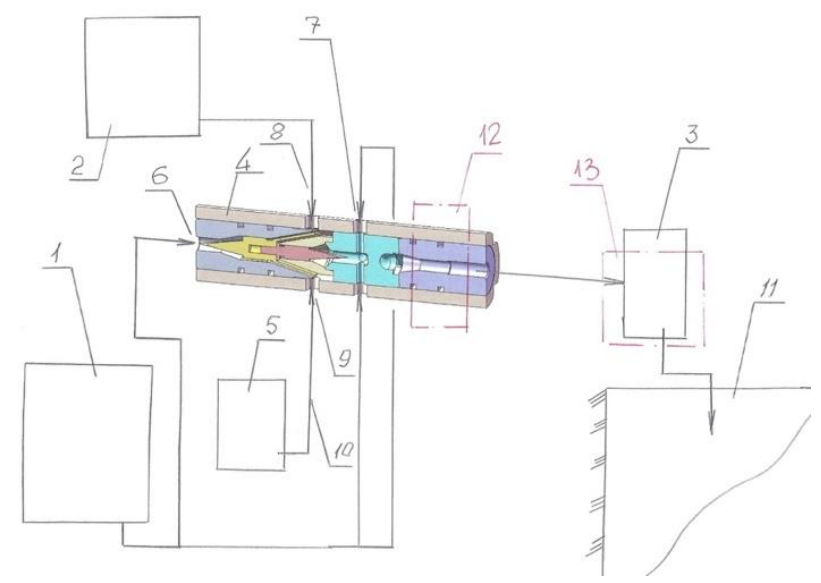

Fig. 3 - provides the combined system of innovative composite fuels preparation at gas stations having the elements of dynamic influence on the composite fuel components as a visual guide of the variants of implementation of the principle of responsiveness in combined infrastructure systems of the smart transport systems (1 - tank with basic fuel; 2 - tank with auxiliary fuel or with composite fuel component; 3 - storage tank with homogenization systems; 4 - fuel composition preparation device; 5 - tank with additional composite fuel component or with water for emulsion preparation; 6 - fuel intake to the device; 7 - fuel intakes to the activating device at homogenization; 8 - fuel component intake; 9 - fuel component intake; 10 - fuel component supply line; 11 - gas station fuel tank; 12 - control area; 13 - control area

16. Principle of partial or excessive action:

- If $100 \%$ of the required effect is difficult to be attained, "a bit less" or "a bit more" must be attained, and the task will become substantially easier.

Actually, for the new innovative product implementing the new process or principle, the definition of the scale of an actual effect is one of the important analytical tasks, which practically can be solved by means of multiple computer models and their modifications that take into account all aspects of the new design.

Computer modeling in such case must take into account all results of preliminary and control tests of the innovative product as well as the results of qualification tests of analogue products.

17. Principle of transition into another dimension: 
- Difficulties related to traveling (or placing) of the object by the line are eliminated, if the object gets an opportunity to travel in two dimensions (i.e. on the surface). Actually, the tasks related to traveling (or moving) of the object in one plane are eliminated when going to the 3 dimension space;

- To use a multi-storied construction of objects instead of the one-storied one;

- To tilt the object or to place it sidewise;

- To use the opposite side of the area;

- To use optical flows falling on the neighboring area or opposite side of the area in question.

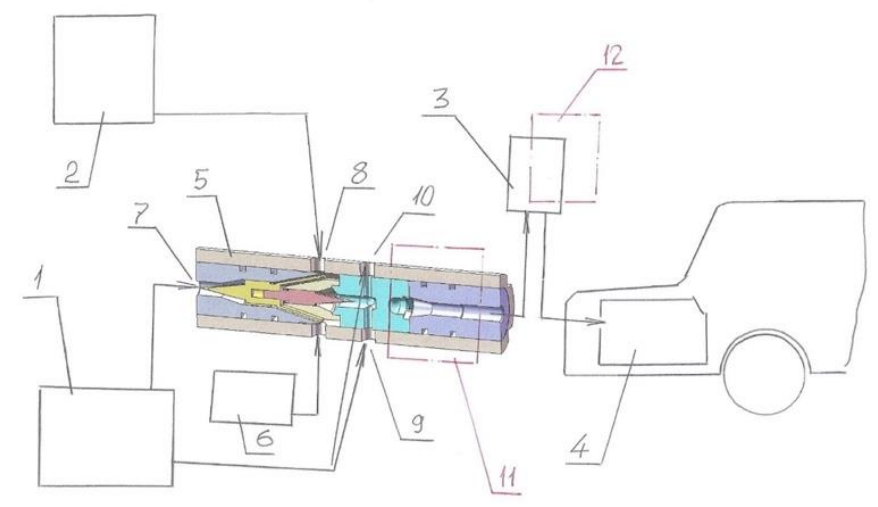

Fig. 4 - provides the combined preparation system at gas stations and transfer of composite fuels or emulsions to the vehicle or another transport means tank or homogenization of fuel before supplying it to the transport means tank (1 - tank with basic fuel; 2 - tank with fuel component; 3 - storage tank before supplying the fuel or composite fuel to the vehicle or another transport means tank; 4 - fuel tank or a vehicle or another transport means; 5 - composite fuel activation device or composite fuel online homogenization device or fuel emulsion preparation device; 6 - tank with auxiliary composite fuel component; 7 - fuel intake to the device; 8 - intake of composite fuel components to the device; 9 intake of composite fuel components to the device; 10 - intake of composite fuel components to the device; 11 - control area; 12 - control area

18. Usage of mechanical oscillations:

- To take the object to the oscillating motion;

- To increase its frequency (up to ultrasonic), if it exists;

- To use the resonance frequency;

- To use piezoelectric vibrators instead of mechanical ones;

- To use ultrasonic oscillations in combination with electromagnetic fields. 


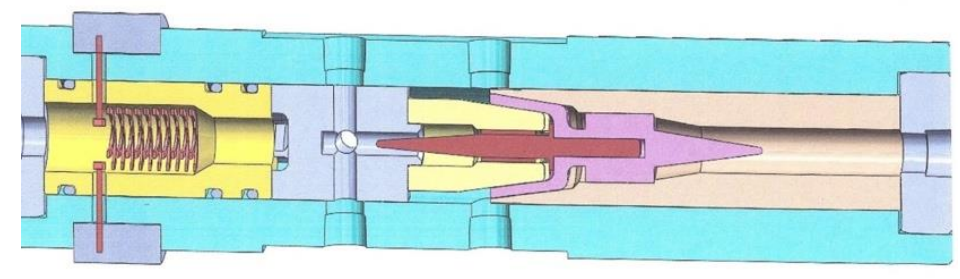

Fig. 5 - provides the device in section for activating the composite fuel with installed resonance sensor in the exit section adjusted for the certain system of parameters allowing to monitor the proportions between the composite fuel components or fuel emulsion

19. Principle of intermittent action:

- To proceed from continuous operation to intermittent (impulse) operation;

- To change the action periodicity, if it is performed;

- To use pauses among impulses for another action.

The composite fuel activation device to the same extent can operate in any regime, because there are no movable parts in it, and it is always ready for action (this is fully related to the following two principles).

20. Principle of efficiency continuity:

- To perform operation continuously (all object parts must continuously operate under full load).

21. Principle of breakthrough:

- To perform the process or its separate stages (for example, harmful or dangerous) at a high speed.

22. Principle of converting harm into benefit:

- To use harmful factors (in particular, harmful effect of the medium) in order to obtain a positive effect;

- To eliminate a harmful factor by means of combining with other harmful factors;

- To enhance a harmful factor to the extent becoming not harmful. 


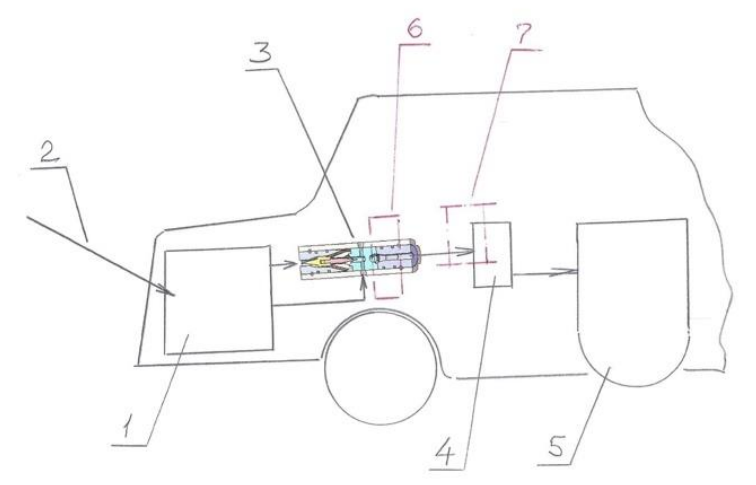

Fig. 6 - provides the process of the fundamental change in the arrangement of the transport means allowing to convert the harm of the necessity to increase the number of units and parts into benefit - the transformation of the transport means in full concordance with the principles of the smart transport means ( 1 - fuel tank; 2 - intake of fuel composition into fuel tank; 3 - composite fuel complex activation device; 4 - high pressure pump; 5 - transport means engine)

23. Principle of feedback:

- $\quad$ To introduce the feedback;

- To change the feedback, if it exists.

The stated principle nowadays is expressly implemented in control systems, including the smart transport means. Besides, it relates to the following three principles.

24. Principle of mediator:

- To use an intermediate object transmitting or transferring the action;

- To connect temporary another (easily removable) object to the object.

25. Principle of self-service:

- The object must be self-serviced performing auxiliary and repair operations;

- To use waste (energy, material).

26. Principle of copying:

- To use simplified and cheap copies instead of the inaccessible, complex, expensive, inconvenient or fragile object;

- To substitute the object or the system of objects by their optical copies (illustrations). To use the scale conversion (increasing or decreasing of a copy);

- To proceed to infrared and ultraviolet copies, if the visible optical copies are used.

27. Cheap fragility instead of expensive durability

- To substitute an expensive object by a set of cheap objects renouncing some properties (for example, durability). 
Long terms of the test operation of fuel and composite fuel activation devices demonstrated that the chosen variant of the design and the determining specifications of the device provide high durability with the optimal cost. This certainly depends upon the factor of the specifications, that determines the possibility of implementation of the production cycle for the device without using traveling parts.

28. Change of the mechanical system:

- To substitute the mechanical scheme by an optical, acoustic or odor one;

- To use electric, magnetic or electromagnetic fields for interaction with the object;

- To proceed from immovable fields to traveling ones, from fixed to changing-with-time ones, from nonstructural to those possessing a definite structure;

- $\quad$ To use the fields combined with ferromagnetic particles.

It is a common knowledge that the most reliable objects are those having no traveling parts. Since one of the factors of the specifications of the fuel and component fuel activation device is absence of traveling parts, the principles of designing, manufacturing and applying of such devices can be adopted as corresponding to the general principle - substitution of the mechanical system.

29. Usage of pneumatic constructions and hydraulic constructions:

- To use gaseous and liquid parts of the object instead of solid ones;

- To use electric, magnetic or electromagnetic fields for interaction with the object: inflatable and hydroinflatable, inflatable cushion, hydrostatic and hydroreactive.

30. Usage of flexible shells and thin films:

- To use flexible shells and thin films instead of regular constructions;

- To isolate the object from the outer medium using flexible shells and thin films.

Thin-filmed micro-assemblies in the microelectronics can be given as an example, using which the response speed got substantially increased, and such increase in the response speed allowed proceeding to smart systems, including smart transport means and smart transport systems, in particular.

31. Usage of porous materials:

- To design the object porous or to use additional porous elements (inserts, covering, etc.);

- To fill in the pores with a substance in advance, if the object is already designed as porous. 
The appearance of composite materials, including those with the pseudo-porous structure allowed at the designing stage to clearly forecast and model the role of porosity or pseudo-porosity in creating the specifications and outcome parameters of the designed device or product.

The appearance of new products possessing unusual properties and characteristics is very frequently explained by the use of porous or pseudo-porous materials and components.

32. Principle of changing the coloration:

- To change the coloration of the object or outer medium;

- To change the degree of transparency of the object or outer medium.

Contemporary computer designing methods allow selecting the variants of coloration and changing the degree of transparency during designing or computer modeling.

33. Principle of homogeneity:

- The objects interacting with the object in question must be designed from the same material (or close to it by properties).

Homogenization of composite fuels or other equivalent materials practically corresponds to the principle of homogeneity.

34. Principle of rejection and regeneration of parts:

- A part of the object, having fulfilled its purpose or became unnecessary, must be rejected (dissolved, evaporated, etc.) or modified immediately during the work;

- Consumable parts of the object must be renovated immediately during the work.

The methods and systems of aerodynamic and hydrodynamic mixture, dynamic preparation of the emulsion and online homogenization are uncharacteristic for this principle, because its basis concludes in the principle of absence of any processes of regeneration and rejection of exhausted parts.

A profound analytical search must be performed for the stated new principles to be qualified in terms of reemulsification and reblending.

35. Change of physical and chemical parameters of the object:

- To change the aggregate condition of the object;

- To change the concentration or consistency;

- To change the flexibility rate;

- To change the temperature. 
This principle in different variants is used in composite fuel and fuel emulsion preparation processes and implemented in homogenization processes.

This principle can be studied wider, if taking into account the fact that the formation of fuel capsules creates new properties for the composite fuel, and the formation of the composite fuel with simultaneous foaming by means of the foam generator creates the compressible liquid effect instead of incompressible liquid.

The same principle is directly related to the following principles of using phase transitions and thermal expansion.

36. Usage of phase transition:

- To use the phenomena appearing at phase transitions, for example, the change in volume, heat generation or absorption, etc.

37. Usage of thermal expansion:

- To use thermal expansion (or compression) of materials;

- To use several materials with various thermal expansion coefficients.

38. Usage of strong oxidants:

- To substitute regular air by enriched air;

- To substitute enriched air by oxygen;

- To use ozonized oxygen;

- To substitute ozonized oxygen (or ionized) by ozone.

This principle is applicable only to the chemical and electrochemical treatment processes. It can also be used in transport systems where the fuel is activated in foam generators.

39. Usage of inert medium:

- To substitute the regular medium by the inert one;

- To conduct the process in vacuum.

Taking into account today's innovative practice, this principle is implemented in the framework of standard computer modeling, and with the correct posing of questions and ideal terms of reference for the corresponding models of the innovative system, the process can be performed much more exactly. And upon its fulfillment it is rather real to obtain the information allowing to open or to predict new variants and new directions with a higher level of novelty and efficiency. 
40. Usage of composition materials:

- To proceed from homogeneous materials to composition ones.

As known, the substitution of one material is not admitted by another invention. The composition material in itself is the topic or object of the original invention, but it happens very frequently when a regular construction material is substituted by a composite changing the properties and possibilities of the product so that the product with composites become an absolutely new, unknown before product, possessing new functions and unusual specifications. Certainly, in order to make such substitution the scope of work must be done comparable with designing an absolutely new technology or a fundamentally new product. And this can be done only by the companies having powerful research units.

Not only composite construction materials but also consumables, for example, fuel in the form of emulsion or mixture are used in smart transport systems as composition materials.

The homogenized fuel with the equivalent viscosity level both in the flow center in the pipeline and in the flow periphery in the pipeline is also the material possessing the composite properties.

Hence, the intake to the device fuel system for online homogenization or online emulsion preparation device, or online fuel blend preparation device are the equivalent of using composite materials, and provide the corresponding effect in the conditions of smart transport.

In the course of situation analysis, we are going back to the TIPS and AIPS tools created for overcoming such type and such complexity of contradiction complexes.

In the Theory of Inventive Problem Solving there is a special software designed for solving complex tasks. The software breaks down the solving process into 50 subsequent steps. The software is provided with special steps helping to overcome the psychological inertia. The software possesses rich data support too. The program is called AIPS, Algorithm for Inventive Problem Solving.

Initially, the "invention technique" was imagined as a code of rules, for example: "To solve the task means to find and to overcome a technical contradiction" or "the stronger solving of the task, the lower consumptions in material, energy, space, time". Several standard techniques were included into the "invention technique": splitting, combining, inverting, changing the aggregate state, substituting the mechanical scheme by the chemical one, etc. 
The information about the work of great inventors, proper inventive practice, materials on the engineering history served as the main source for revealing the rules and techniques.

By the middle of the 50ies the persuasion had been developed and strengthened that the inventors, even the sturdy ones, operated using the ineffective trial and error method and, therefore, the ambition to reveal and to use the "secrets of art" is prospectless.

A fundamentally new "invention technique" must be developed which is based on using the objective laws of development of engineering systems. These laws can be revealed by the systematic analysis of large files of patent information.

By the end of the 50ies of the previous century it was clear that: the "invention technique" must include not only AIPS, but also the section about the laws of development of engineering systems and ever-expanding information fund. The "invention technique" had to give way to the "science of invention". This idea encountered strong resistance. The "invention technique" was treated as something more or less tolerable: ultimately, those are helpful recommendations based on studying the inventors' experience, not obviously overthrowing the "sacred" notions. The "science of invention" targeted the "sacred" negating the exceptionalism of great inventors, touched upon traditional beliefs on unapprehensiveness of the creative process. The "invention technique" helped to "get lighted up" - the "science of invention" negated all old fashion technology, negated the inborn aptitudes.

The software becomes harder and more definite through the years. In the course of the analysis the operative area and conflicting requirements, lodged to it, are defined (prototype of FP). The RVS operator was introduced. The TP elimination table was finalized, the list of approaches was renewed (40 from the beginning, 50 after that). Instructions for making steps, notes, examples were introduced. The main operators form the system - the interrelation between the steps got strengthened, a new part appeared - preliminary evaluation of the found idea.

The situation has drastically changed through the years with the appearance of the processor equipment making it necessary to treat the reliability notion absolutely in a different way, because the rigid, mechanical conception of reliability gave way to more flexible one - by virtue of more clear management process and working cycle while using analytical and control opportunities of the processor equipment. 
The following main directions in the evolutionary development of TIPS and AIPS can be distinguished in terms of synthesis and modification of comprehensive engineering solutions, where the integrative reliability of the system is one of the fundamental base values: 1. Traditional for the evolution of AIPS - general increase in the algorithmization degree due to more complete and more profound usage of objective laws of development of engineering systems and processor systems.

2. Substantial strengthening of the "bridge" between the physical contradiction and the method of solving it, including the one based on using composite materials and the latest developments in digital technology.

3. Enhancing the information fund, strengthening the interactions between AIPS and standards, including the combination with operative production standards and environmental regulations, which requirements and limitations contradict the traditional economic regulations.

4. The advantageous development of the second half of AIPS (development and use of the found idea) into a standalone algorithm having the components of "Program, system and method" or "Apparatus, device, system, program and associated method".

5. Designing of a new initial part (or a separate algorithm) for discovering new composition and integrative tasks.

6. Enhancing the general educational function. AIPS must develop the skills of strong, comprehensive thinking more actively.

7. Gradual increase of the universalism in the course of creating the composition model of the apparatus or process closely related to the software and processor equipment.

\section{References and patent materials}

1. Provisional patent USA No. US 62/622,143, Feb. 23, 2018. Apparatus, system and method for producing emulsion with re-emulsification properties / Valentin Korobov;

2. Provisional patent USA No. US 62/694,450, Jul. 26, 2018. Engine with integrated mixing, emulsification, homogenization and stabilization technology in real time / Valentin Korobov;

3. Patent application USA No. 20140232021, Aug. 21, 2014. Fluid composite, device for producing thereof and system of use / David Livshits;

4. V.V. Korobov. Usage of analytical TIPS and AIPS tools for applying for the USA patent. Technical guide. -2018.

5. V.Korobov. Adaptation of TIPS and AIPS to the technologies and design of Smart Transportation. LAP Lambert Academic Publishing. - 2019. 